\title{
Independent component analysis in the detection and correction of physiological artifacts in fMRI
}

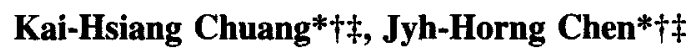 \\ Department of Electrical Engineering, Interdisciplinary MR Lab, \\ National Taiwan University, Taiwan, ROC
}

\begin{abstract}
Introduction
Motion artifact is one of the most serious problems in functional MRI (fMRI). Physiological motions, especially respiration and heartbeat, are the important noise sources when the head does not move at all. Current correction methods require external monitoring of physiological cycles, $\mathrm{k}$-space data, or specialized pulse sequences [1-3]. Recently, an image-based method is proposed without these limitations [4]. Independent component analysis (ICA) is an emerging analysis method that can detect various signal and noise [5, 6]. Here, we evaluate its capability in detecting and correcting the physiological motion artifacts.
\end{abstract}

\section{Methods}

Resting state and visual fMRI experiments were performed on a Bruker $3 \mathrm{~T}$ scanner (Ettlingen, Germany). 400 single-slice $64 \times 64$ EPI scans were obtained from 4 subjects (men; ages 23-30) with parameters: TR/ $\mathrm{TE}=250 / 35 \mathrm{~ms}, \mathrm{FOV}=25 \mathrm{~cm}$, thickness $=5 \mathrm{~mm}$. Visual stimulation was delivered by a pair of red LED goggles flashing at $8 \mathrm{~Hz}$. Respiratory and cardiac cycles were recorded simultaneously by pressure belt and EKG leads.

The data was separated by spatial ICA into 60 components with data dimension reduced by principal component analysis. Several ICA components showing significant physiological cycles were identified. Two correction approaches by ICA were studied. One was removing the components containing the artifacts directly. The other was applying notch filters centered at the identified physiological frequencies to the column vectors of the mixing matrix (i.e. the ICA waveforms). Then artifact-removed data was formed by multiplying the mixing matrix by the component images. The effect was compared with an image-based correction method [3] and notch filtering. The artifacts-removed activation maps were analyzed by correlation analysis with threshold $=0.5[7]$.

\section{Results}

Fig. 1 shows the spectrum of a pixel time-course. Although removing ICA components directly could suppress the "noises" in widespread frequency bands, a cardiac-related fluctuation was increased. Filtering the ICA waveforms avoided this problem. Compared with the image-based correction method and notch filtering, ICA seemed to remove something other than the physiological artifacts. Compared with the activation maps corrected by the image-based method, removing the ICA components directly increased activated areas too much (Fig. 2). Using the modified approach, the activated regions became similar to the results of the image-based method.
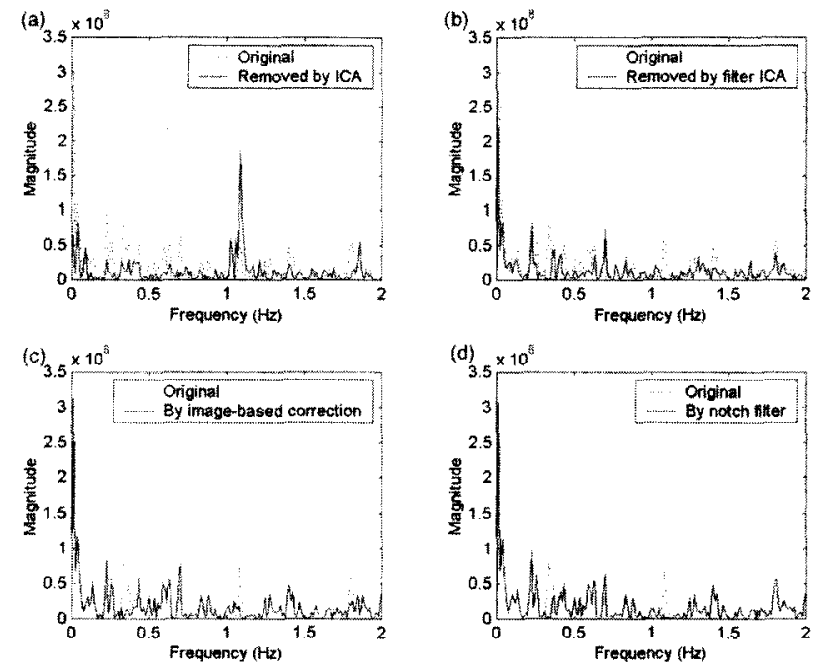

Figure 1. Spectrums of one pixel time-course before and after corrected by (a) removing ICA compoents directly, (b) filtering the ICA waveforms, (c) an image-based correction method, and (d) notch filters.
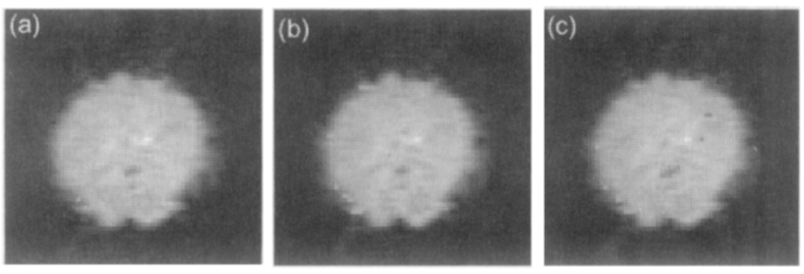

Figure 2. Activation maps after processed by (a) image-based correction, (b) removing ICA components directly, and (c) filtering ICA waveforms

\section{Discussion}

Since a component image has contribution to every pixel in the data, removing the components showing significant artifacts in some of the pixels will affect the signals of other pixels. In addition, ICA may mix some other signal or noise processes with physiological artifacts. Thus signal fluctuation could be reduced significantly by removing other noise sources. However, some meaningful signals could be removed as well. Using the modified approach could remove the physiological artifacts more precisely and make the activation maps closer to the results processed by conventional correction method. Currently, the effects on multi-slice data are under investigation.

\section{References}

[1] Hu X et al.. Magn Reson Med 1995; 34:201-12.

[2] Stenger VA et al., Magn Reson Med 1999; 41:983-91.

[3] Glover GH et al., Magn Reson Med 2000; 44:162-7.

[4] Chuang KH and Chen JH. Proc ESMRMB, 2000.

[5] Bell AJ and Sejnowski TJ. Neural Comput 1995;7:1129-59.

[6] McKeown MJ et al., Hum Brain Mapp 1998:6:160-188.

[7] Bandettini PA et al., Magn Reson Med 1993; 30:161-73. 